\title{
Adaption of cost calculation for modular LBPF machine
}

\author{
Sebastian Dirks 1, Johannes Henrich Schleifenbaum 1,2
}

\begin{abstract}
Methods for cost calculation of Laser-based Additive Manufacturing (LAM) have evolved over the last two decades. Starting from a rapid prototyping manufacturing method, cost calculation over time included more than single part production for small series or mixed batches. New machine developments nowadays aim at large scale production and introduce modular machine designs that give LAM factory operators more decision freedom on machine configurations and expansibility. To leverage this new possibilities, LAM costing methods must be adapted to calculate and visualize the economic consequences of different module configurations.

In this work a modelling architecture of L-PBF machines using virtual modules is presented. Using principles of the industry 4.0 communication standard OPC UA, a virtual machine is set up, parametrized and connected to the AM digital process chain. Two different views on the cost information are used, optimized for different use cases.

First, the product view allows detailed analysis of cost allocation to parts in mixed batches and the layered geometry of the part. Second, the machine view tracks utilization of all machine modules, divided into the states idle, active, off and maintenance. The theoretical Overall Equipment Effectiveness (OEE) is calculated on a module basis, enabling economic analysis and optimization of module composition as well as module improvements and new modular machine constellations. This allows focusing research and development on most valuable improvements. By calculating different scenarios like spare part production, mass production or rapid prototyping, the part portfolio to be manufactured can be considered.
\end{abstract}

\section{Keywords}

Industry 4.0, LAM Costing, modular LAM/L-PBF machine design, digital twin, OPC UA, OEE

\section{Introduction}

Laser-based Additive Manufacturing (LAM) is seeing an ongoing trend towards larger scale, series production systems. Most major market players already offer or plan to offer modular, scalable systems that can be configured by their customers. Examples include the MetalFAB1 by Additive Industries [1], the M400 by EOS Electro Optical Systems [2], the M Line Factory by GE Additive [3], the SLM 500 by SLM Solutions Group AG [4] and the TruPrint 3000 by TRUMPF Laser- und Systemtechnik GmbH [5].

1 Chair for Digital Additive Production at RWTH Aachen University

2 Fraunhofer Institute for Laser Technology ILT 
These modular systems are configurable by the AM factory operator. They consist of machine modules and auxiliary systems (e.g. sieving and unpacking stations) that can be combined in various constellations. They are scalable by adding modules and can be ordered in different expansion levels (e.g. the MetalFAB1 offers optic modules with 1-4 laser units). These systems follow different machine concepts, and the economic viability for the use case of each individual company is not easily defined. On the one hand the flexibility of the concepts increases possible combinations and thus AM investment planning complexity. On the other hand the economic benefit strongly depends on the geometry and constellation of the part portfolio to be manufactured on this machines.

In this paper a new approach in LAM cost calculation that is adapted to this new machine concepts is presented. A virtual machine consisting of modules is integrated into the digital process chain of AM manufacturing, enabling modelling of modular concepts and calculation of machine viability on a scenario basis as well as analyzing the Overall Equipment Effectiveness (OEE) on a module basis.

\section{Short AM Cost Calculation History}

Cost calculation for Laser-based Additive Manufacturing (LAM) has evolved over time, just like the production method itself. Additive manufacturing has its roots in the rapid prototyping of parts, where builds consisted mostly of single parts. This short overview highlights some cost calculation models that have gained some traction in the literature [6], and lists them in chronological order of their publishing dates.

The early cost model by Gibson et al. [7] uses an analytical approach. This model calculates the build costs of a single part by calculating input parameters from the geometry of the part. The cross section area of the part in each layer is the main input, the processing time is estimated with a simple model of equidistantly spaced paths. The part volume determines the material costs. Support volume is estimated by the area of downwards-facing surfaces and calculated in the same way.

The activity based costing approach by Hopkinson and Dickens [8] focusses on small series production. It includes indirect costs into the calculation, like cost for build preparation, set-up time and post-processing of parts. The model assumes a production of the same part for one year with the maximum possible parts per build.

Rickenbacher et al. [9] use a cost model that includes building of different part geometries in one job. The model includes seven steps: Cost for preparing geometry data, cost for the build job assembly, machine set up costs, cost of building the part, cost for removing the part from the L-PBF machine, cost for separating the parts form the substrate plate and costs for post processing. The build time is estimated with the following regression formula:

$$
\underset{i}{\boldsymbol{L}} T_{\text {Build }}\left(P_{i}\right)=a_{0}+a_{1} * N_{L}+a_{2} * V_{\text {tot }}+a_{3} * S_{\text {supp }}+a_{t o t} * \underset{i}{\boldsymbol{L}} N_{i}+a_{s} * S_{\text {tot }}
$$

The building time $T_{\text {Build }}$ for the part with $i_{\text {th }}$ geometry is calculated with the regression coefficients ao to $a_{s}$. Input parameters are the number of layers $N_{L}$, the total volume of the build job $V_{t o t}$, the total surface area of the support structures $S_{\text {supp }}$ tot the quantity of parts with $i_{\text {th }}$ geometry $N_{i}$ and the total surface area of the build job $S_{\text {tot. }}$. The regression coefficients are derived from 24 build jobs on one machine. az to $a_{s}$ are used as well to allocate the total build time to each part, while $a_{o}$ and $a_{I}$ are distributed among all parts equally. The layer dependent 
$a_{I}$ is only distributed among parts that still have geometry in the layer, causing parts with less height that would allow the job to finish earlier to be priced fairly.

Recent online cost estimation tools as described e.g. by Rudolph et. al. [10,11] focus on the fast, fully automated calculation of an estimated price based on an uploaded STL part geometry. Similar tools can be found on most 3D printing service provider platforms. The described model uses similar formulas as Rickenbacher et al. using build height, volume and surface area as inputs. The performance of the estimation shows a mean absolute percentage error (MAPE) of $8.2 \%$ on the build time in part screening.

None of the reviewed cost models takes scalable, modular multi-laser systems into account. Most cost models are not considering mixed batches of e.g. spare parts that are an important use case of additive manufacturing (AM). The allocation of costs to parts is based only on substitute quantities like volume, not on the time the machine spends to produce geometry belonging to this part. Modular machine concepts can't be represented with the models. The build time estimation precision presented in [10] shows a MAPE of $8,2 \%$. The estimation is only valid for one machine, and additionally build job length data from this machine must be available to conduct the regression. To overcome these shortcomings, a new modular benchmarking approach on the basis of a virtual machine module architecture is presented in this paper.

\section{Basic Software Architecture Model}

The proposed benchmarking software acts as a machine emulator that gets fully integrated into the digital L-PBF process chain (Fig. 1). It uses the same interfaces as real additive manufacturing machines. The software reads different formats of the layered 2,5D geometry data that controls the galvanometer scanner and replaces the real machine in the "execution of vectors" step. This data is created from 3D modelling data in the "slicing" process step. Opposed to analytic cost models, the use of the full set of machine control data enables a highly precise time estimation. The knowledge of the process, material and machine dependent parameters is essential for the model. And even more importantly, all geometry dependent factors in the digital process chain are included in the calculation. This allows the benchmarking of every single software component in the digital process chain with different input scenarios (e.g. mixed batch spare parts, small series or large parts). Exemplary geometry dependent use cases are the benchmarking of different manufacturing parameter sets, the performance of multi-laser scan field allocation algorithms or the cost effectiveness of different job nesting algorithms.

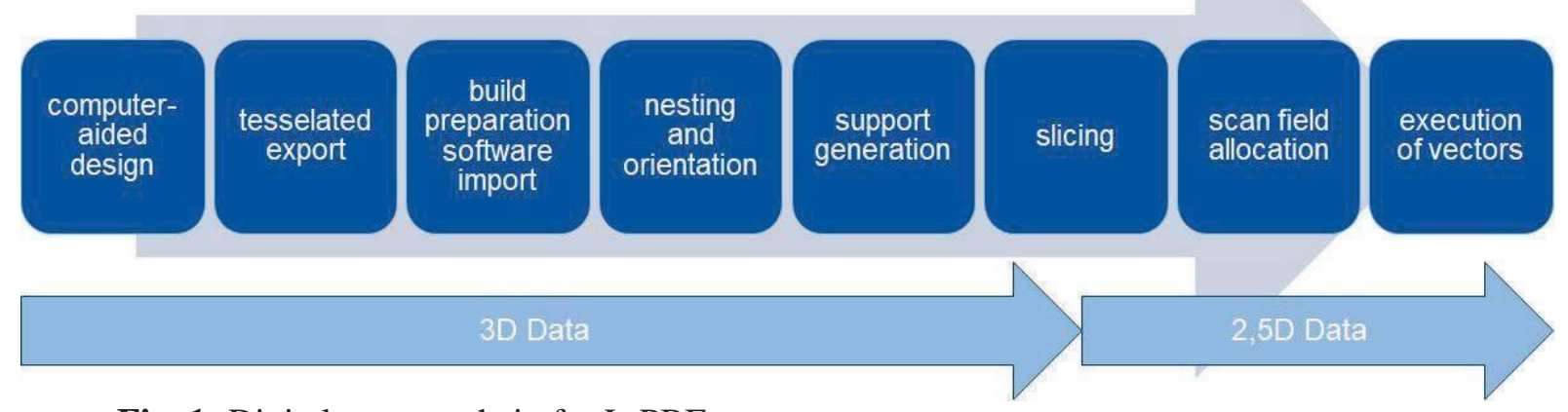

Fig. 1: Digital process chain for L-PBF 
The downside of using the layered data is that it usually has to be prepared manually, which makes the approach less viable for a quick automatic quoting based on a 3D STL input. An automatic slicing of the input is possible, but may lower the precision depending on the discrepancies between automated digital process chain and the manual chain the part is following in production.

The results of the cost analysis as well as the parametrizing of the machine emulator is conducted via an self-describing Open Platform Communications Unified Architecture (OPC UA) interface. OPC UA is gaining traction as an Industry 4.0 standard for the communication between manufacturing machines in the shop floor and higher-level control systems [12]. Including this interface enables the future integration of the emulator into OPC UA based manufacturing execution systems (MES). All functions of the emulator are called via OPC UA method calls. The results of the benchmarks are summarized into two different views: The machine view, which shows the usage of every module of the machine, and the product view, which distributes the costs of the build job among the parts printed in the job ( Fig. 2).

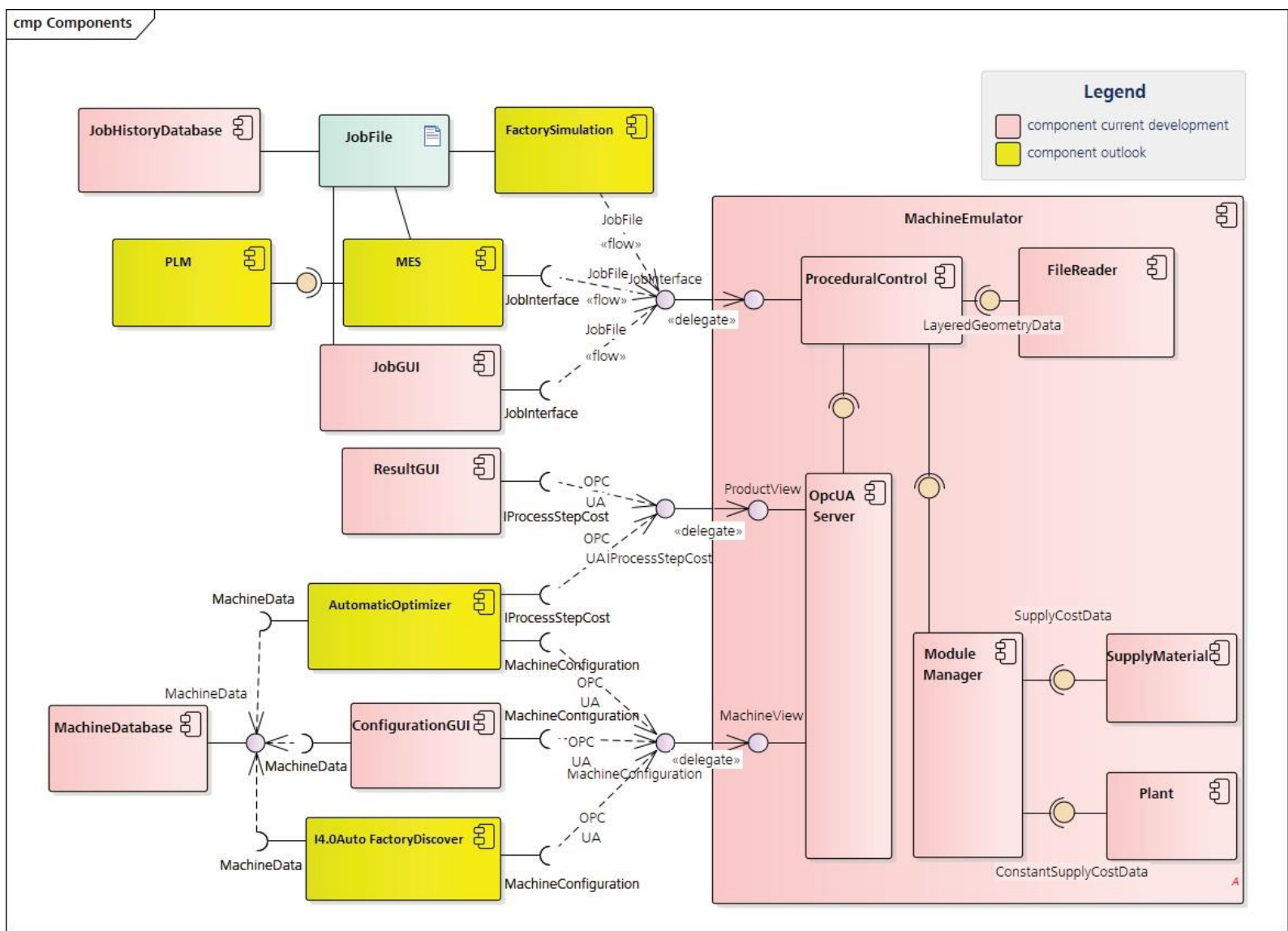

Fig. 2 Basic architecture of the machine emulator (UML component diagram)

\section{Machine view}

The machine view enables the detailed analysis of the overall equipment effectiveness of a LAM machine on the basis of a module concept. The primary use case of this view is the benchmarking and improvement of machine concepts by identifying cost drivers among the modules. It is also possible to evaluate the consequences of specific machine improvements. 
This includes replacing parameters like adapting the coater speed, but also highly geometry dependent improvements like non-state of the art scanning strategies. The primary user of this view is the machine manufacturer, although it can be used by advanced customers with in-depth process know-how as well.

\subsection{Modules}

Every component of the machine as well as the whole machine is considered a module. Each module has its own state machine with the four basic states active, idle, off and maintenance. The modules measure their activity time in each state, which enables analysis of the OEE on a module basis. E.g. the overall laser on-time of each laser in a multi laser machine can be tracked. The states are associated with energy consumption, e.g. the laser module keeps track of its time in the active state with the energy consumption depending on the laser power defined by the current process parameters. The module types implemented in the module framework can be found in Fig. 3. The types have been derived from the state of the art in modular machine concepts [1$5]$.

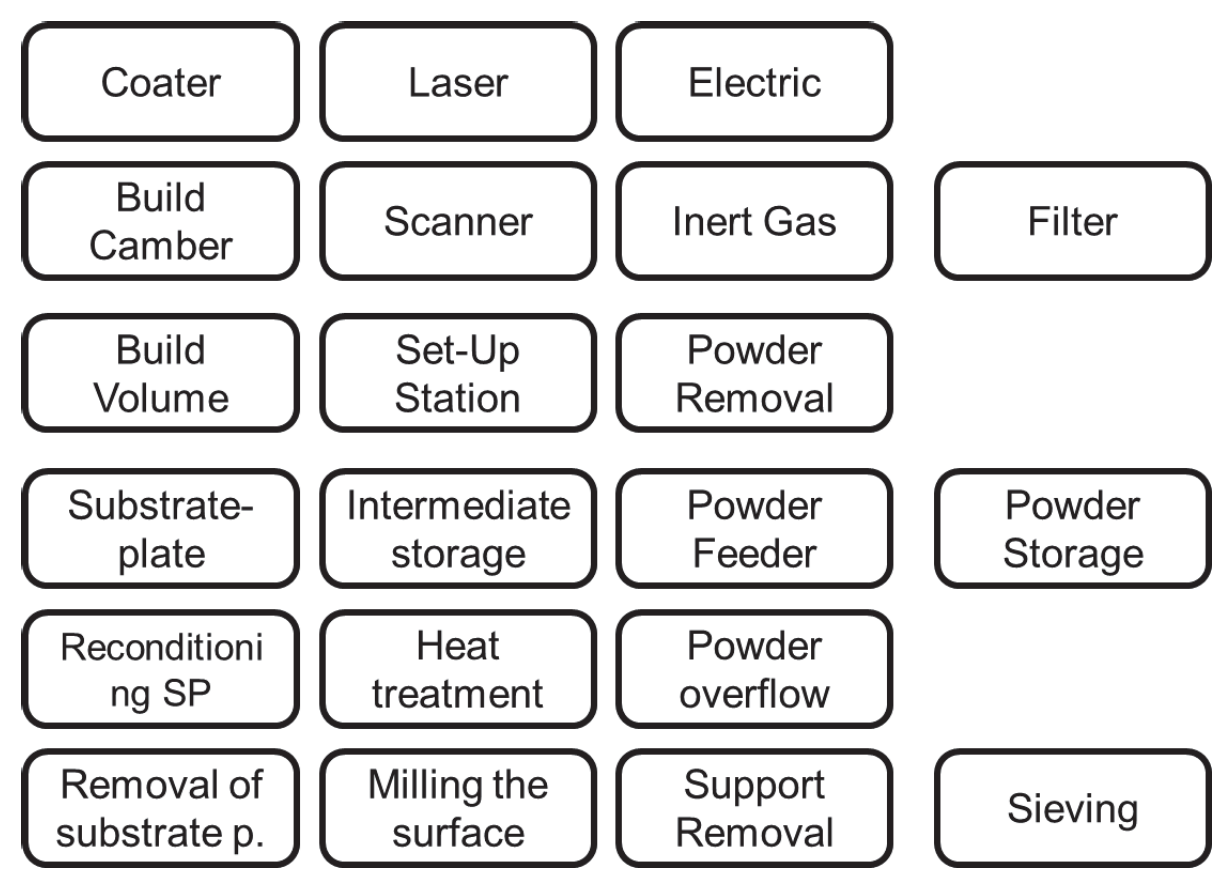

Fig. 3: module types of the simulator

The OEE of different module types is influenced by the geometry of the manufactured parts in varying degrees. For the scanners and their associated lasers in a multi-laser machine this influence is the strongest, as they execute the vectors that form the geometry. The scanners have to be synchronized to each other before recoating for the next layer, which can result in idle times when the vectors are not evenly allocated to every scanner. The coater module is influenced significantly as well, its usage depends on the part height, layer thickness as well as the ratio between exposure and coating time associated with the filling degree of the layer cross sections.

All other modules are influenced indirectly by the overall build time of the jobs, since most of the post processing modules (e.g. heat treatment, removal of parts, surface milling) are 
shared by multiple build chambers in most machine concepts to increase their utilization ratio. Modules associated with the powder cycle (powder storage, powder feed and overflow, sieving, filter) are influenced by the volume of the parts that are build.

\subsection{Services}

To obtain flexibility in implementing the modules, the software architecture uses the concept of services. Each module implements services associated with functions in the procedural control of the LAM build process. The services are the connection between the digital process chain and the module implementation. Procedural controls are not unique, different variants are possible that offer different service slots. As an example, a procedural control that is multi-laser capable needs to take the scan field locations and overlap of different laser-scanner units into account and has to allocate vectors to the lasers.

Different variants of modules can implement the same service, being controlled by the same interface but with different implementations. This offers flexibility in benchmarking new module concepts, where a calculation needs to be integrated into the virtual module.

The basic procedural control implemented is oriented on the classical L-PBF process cycle [13]. It controls the modules coater, build chamber with inert atmosphere flooding and preheater, powder feed and unload, platform lift and multiple laser scanner units. It receives the 2,5D layered geometry data and executes the L-PBF process by flooding the build chamber and concurrently preheating. For each layer powder is feed and recoated, then the laser-scanner units are executing the exposure. After exposure, the platform is lowered by the platform lift and the cycle starts anew until all layers are finished. After the build job is finished, the inert atmosphere is released and the powder and substrate plate is unloaded.

The user of the software can create virtual machines using a module tree. Child modules are dependent on their parent and will be synced to a timestamp when their parent is synced, e.g. a substrate plate in a build volume will be used exactly as long as the build volume itself. The modules in the tree can then be connected to a service interfaces they implement called by the procedural control. All this actions are realized using OPC UA method calls.

\subsection{Parameter Sets}

Each module has a specific set of parameters that define its performance (e.g. the coating speed of the coater, the delays the scanner uses etc.). The software concepts allows for the creation of multiple parameter sets independent of modules and associating parameter sets of the correct type to each module. Modules can share the same parameter set, e.g. one set of scanning parameters for every scanner in a multi-laser machine. Parameter sets are published in the OPC UA interface and can be set by OPC UA read and write functions.

\section{Product view}

The product view is a different perspective on the same data calculated in the machine modules. Its primary user is the AM factory operator. In this view the costs of the build job are allocated to the parts of the job. In contrast to a simple allocation using the volume, surface or other simple variables to split the costs [9], the allocation is based on the sum of the exact time 
each vector block in the layered geometry data has taken for execution on the machine. All vector blocks are associated to one 3D part only by definition. Times per layer which are not associated to one part, mainly coating and platform lowering, are split evenly upon all parts that have geometry in the layer. This results in the same cost split used by Rickenbacher et al. [9].

Times associated to the build job as a whole, e.g. preheating, inerting the atmosphere or unloading the substrate plate are evenly distributed among all parts in the job.

\section{Verification of the model}

The most crucial component for the verification of the model is the calculation of the exposure time. This time component is heavily influenced by the part geometry and takes most of the manufacturing time of an L-PBF machine. Thus the galvanometer scanner model is the most complex in all the modules. All parameters set in the virtual scanner have to be set correctly. Parameters included in the model calculation are jump speed, delay times (jump delay, mark delay, polygon delay, laser on- and off-delay), sky writing pre- and post run including geometric vector extension, jump start and end point recalculation as well as limit angle between vectors for applying sky writing. The implementation includes multi-laser machines, using multiple scanner modules to execute the exposure in parallel and syncing all scanners correctly to the longest exposure execution.

The calculated times are compared to the timestamps of the machine log file on a layer basis for nine real-life build jobs on a dual laser machine. The overall results of the verification are shown in Fig. 4. The deviation is between $-0.42 \%$ and $0.67 \%$, resulting in variations form -7 to +6 minutes in the predicted build time. This results in a MAPE of $0.28 \%$.

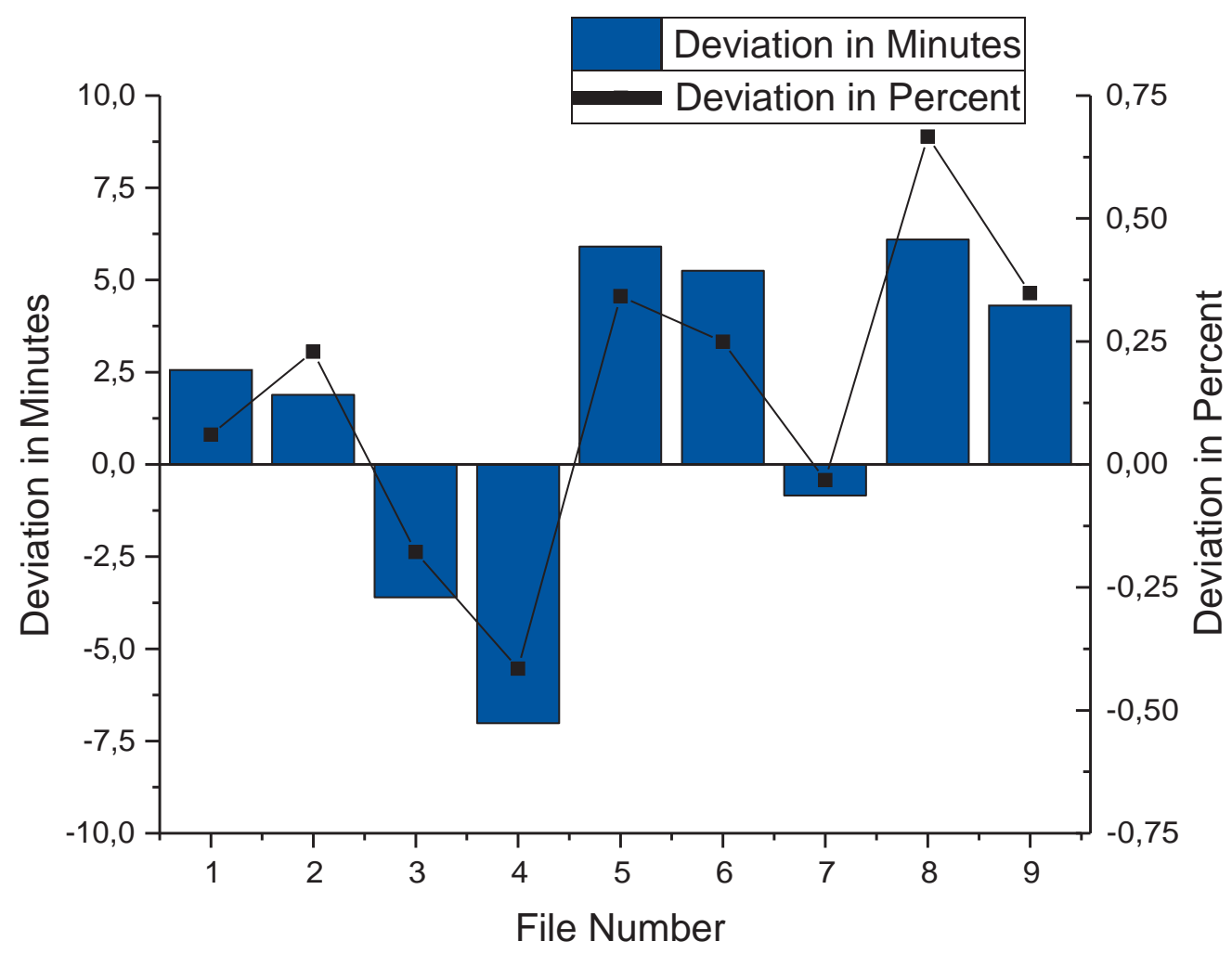

Fig. 4 Deviation between calculated and real build time in percent and minutes per build job file 
The average deviation of the exposure time per layer from the machine log for the same files is shown in Fig. 5. The absolute deviations are below $0.2 \%$ on average for every single layer of the file, and also show a low standard deviation below $1 \%$, showing the accuracy of the model is geometry independent and thus able to also predict unknown or unusual build jobs with the same precision.

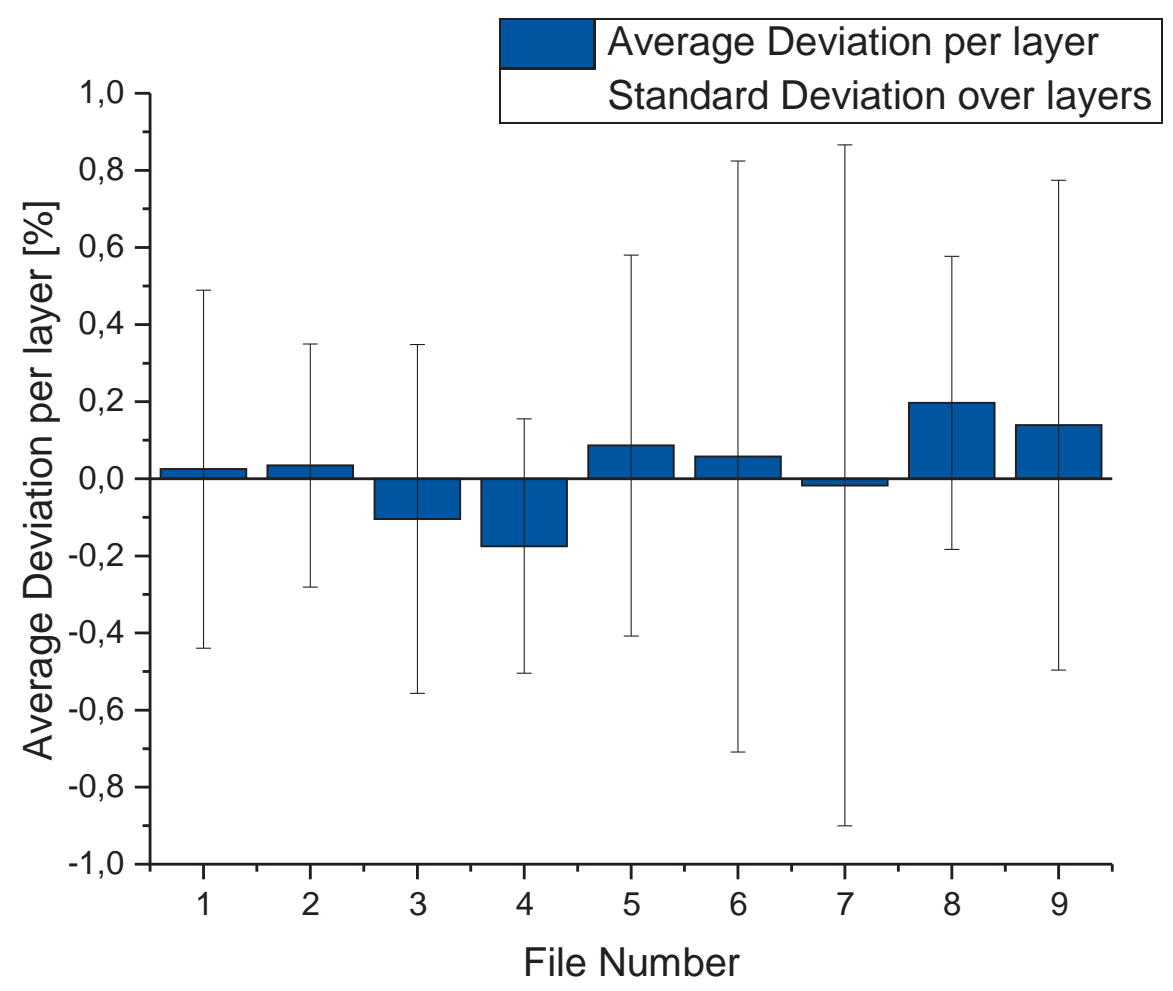

Fig. 5 Layer-wise average and standard deviation between simulated and real exposure time per build job file

\section{Summary and outlook}

In this work a new approach on L-PBF cost calculation has been presented. It adapts to the trend of modular AM machines using virtual modules. The shortcomings of past cost calculation methods are analyzed: no support of scalable multi-laser systems, mixed batches are not included and the cost is imprecisely split upon parts in the job.

The virtual module emulator approach uses the full L-PBF digital process chain and replaces the real machine in the "execution of vectors" step by processing layered 2,5D geometry data. Several module types are identified from the state of the art modular systems and implemented in a common framework. A procedural control executes the L-PBF process cycle by calling services. These services are implemented by different module variants, leading to a service-orientated architecture. Modules can be quickly parametrized by using parameter sets independent of modules.

The machine module tree is build up and the parameters are set via an OPC UA interface, enabling integration into industry 4.0 environments. The benchmark results are presented via OPC UA as well, using two different perspectives: The machine view shows the OEE on a module basis, the product view shows the cost split on the parts in the job based on the execution time of the sum of vector blocks of each part. 
The calculated build time of the emulator is verified by comparing it layer-wise to log timestamps of a dual laser L-PBF machine with nine real-life build jobs. The MAPE reaches $0.28 \%$ for the model, a huge improvement over regression models found in literature with 8.2 \% MAPE [10]. The layer-wise analysis shows an average deviation below $0.2 \%$ with a standard deviation below $1 \%$, concluding in a nearly geometry independent prediction of build time.

As the next step, the verified machine model based on the physical components will be used as a basis for simulation studies of various parameter changes in the model and their effect on the cost of building a given part portfolio in a manufacturing scenario. The model will enable the prediction of economic benefits of different machine improvements and will function as a tool to optimize build job nesting. Due to its OPC UA interface it can be integrated into future OPC UA based Manufacturing Execution Systems (MES) to set up a full virtual factory of LPBF machines.

\section{Acknowledgements}

This research has been financed by the Germany ministry of Research and Education in the Digital Photonic Production Direct (13N13710) project as part of the "Research Campus Public-Private Partnership for Innovation" initiative.

\section{References}

[1] Additive Industries B.V.

[2] EOS Electro Optical Systems, EOS M 400. System zum industriellen 3D Druck von großen Metallteilen im Produktionsumfeld. <https://www.eos.info/systeme_loesungen/metall/systeme_und_zubehoer/eos_m_400>.

[3] GE Additive, Concept Laser M Line Factory. <https://www.ge.com/additive/additivemanufacturing/machines/dmlm-machines/m-line-factory>.

[4] SLM Solutions Group AG, 2018.

[5] TRUMPF Laser- und Systemtechnik GmbH, 2019.

[6] G. Costabile, M. Fera, F. Fruggiero, A. Lambiase and D. Pham, International Journal of Industrial Engineering Computations 8 (2017). doi:10.5267/j.ijiec.2016.9.001.

[7] Gibson, I., Rosen, D. W., and Stucker, B., Additive Manufacturing Technologies: 3D Printing, Rapid Prototyping, and Direct Digital manufacturing, 2nd., Springer, New York, (2015), pp. 1-498.

[8] N. Hopkinson and P. Dickens, Proceedings of the Institution of Mechanical Engineers, Part C (Journal of Mechanical Engineering Science), 2003, Vol.217(1), pp.31-40 217 (1) (2003). doi:10.1243/095440603762554596.

[9] L. Rickenbacher, A. Spierings and K. Wegener, Rapid Prototyping Journal, 19 April 2013, Vol.19(3), pp.208-214 19 (3) (2013). doi:10.1108/13552541311312201.

[1O] J.-P. Rudolph and C. Emmelmann, Procedia CIRP 63, 412-417 (2017). doi:10.1016/j.procir.2017.03.087.

[11] J.-P. Rudolph and C. Emmelmann, Procedia CIRP 67, 185-190 (2018). doi:10.1016/j.procir.2017.12.197.

[12] VDW e.V., vdw-umati. umati-universal machine tool interface OPC UA Companion Specification for machine tools and manufacturing systems to external communication partners. <https://opcfoundation.org/markets-collaboration/umati/>.

[13] W. Meiners, Direktes selektives Laser Sintern einkomponentiger metallischer Werkstoffe, Dissertation, 1999. 